\title{
Additive Manufacturing (AM) Capacitive Acoustic and Ultrasonic Transducers Using a Commercial Direct Light Processing (DLP) Printer
}

\author{
B. Zhu, J. Guerreiro, Member, IEEE, Y. Zhang, B. Tiller and J. F. C. Windmill, Senior Member, IEEE
}

\begin{abstract}
In recent years, there has been increasing interest in using additive manufacturing (3D printing) technology to fabricate sensors and actuators due to rapid prototyping, low-cost manufacturing processes, customized features and the ability to create complex geometries at micrometre scale. State of the art additive manufactured acoustic and ultrasonic transducers show limitations in miniaturization, repeatability (defects) and sensitivity. This new work encompasses the development of a capacitive acoustic and ultrasonic transducer, including its fabrication process using a commercial digital light processing printer and output signal characterization with a custom-made amplification circuit. A set of capacitive acoustic and ultrasonic transducers was fabricated and tested using different diaphragm diameters from $1.8-2.2 \mathrm{~mm}$, for comparison, with central operating frequency between $19-54 \mathrm{kHz}$, respectively. This capacitive transducer design has a receiving sensitivity of up to $0.4 \mathrm{mV} / \mathrm{Pa}$ at its resonant frequency, and a comparison with a commercial reference microphone is provided.
\end{abstract}

Index Terms - Additive Manufacture, AM, 3D printing, Transducer, Sensor, Direct Light Processing, DLP

\section{INTRODUCTION}

Capacitive transducers for acoustic or ultrasonic measurement have been investigated for several decades. Currently, the performance of commercial capacitive transducers has reached a very high standard. However, most companies, for example, the well-known Danish company Brüel \& Kjær (B\&K), are still utilizing a traditional manufacturing technology and manually assembling these transducers from parts, resulting in low production volume and relatively higher cost [1]. Microelectromechanical systems (MEMS) is a more recent manufacturing technology that has commercially grown rapidly during the last two decades and is used in a variety of applications, e.g. mobile devices and wearable devices. MEMS microphones can achieve reasonable sensitivity at low bias voltages due to their narrow air-gaps [2]. Many researchers have proposed several transducer or microphone designs with complex 3D structures in order to explore different ways to enhance their performance such as sensitivity [3] [4], bandwidth [5], [6] and directivity [7]. In recent years, there has

This paragraph of the first footnote will contain the date on which you submitted your paper for review. It will also contain support information, including sponsor and financial support acknowledgment. For example, "This work was supported in part by the U.S. Department of Commerce under Grant BS123456."

B. Zhu, J.Guerreiro, Y Zhang, B. Tiller and J.F.C. Windmill are members of the Centre for Ultrasonic Engineering, Electronic and Electrical Engineering also been increasing interest in using additive manufacturing (AM) technology (also known as 3D printing) in the fabrication of sensors or actuators owing to the possibility to create complex 3D geometries at micrometre-scale using highly flexible polymers. After integrating multiple nano-fillers with the passive base polymer, an AM nano-composite can exhibit different properties including conductivity [8] or piezoelectricity [9]-[11], which allow the transduction of mechanical energy into electrical form. There are many reports of actuators [12], [13] and sensors [10], [14]-[17] fabricated using AM processes, however, various limitations in miniaturization [16], repeatability (defects) [16] and low sensitivity [10], [17] are also reported.

This paper demonstrates a methodology for using a commercial digital light processing (DLP) printer to fabricate capacitive transducers which integrate a custom-made passive polymer with a custom-made conductive polymer. The transducer is small enough to be integrated with surface-mount electronics, and it is tested against a gold-standard reference B\&K microphone, showing comparable electro-acoustic sensitivity and following a predictable fabrication process. Nevertheless, the structure layout presented in this paper is a conventional capacitive transducer with a simple structure as shown in Figure 1. Additionally, this AM framework has the potential to benefit the fabrication of acoustic and ultrasonic diaphragm transducers with more complex geometries such as the ones used in biologically inspired transducer designs [7], [10], [18].

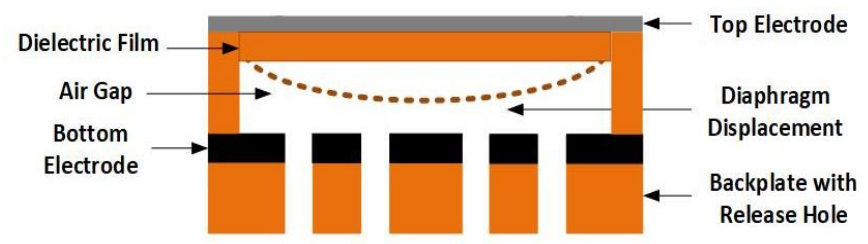

Figure 1. Simplified schematic of a conventional capacitive transducer.

Department, University of Strathclyde, Glasgow, Scotland, UK, G1 1XW (email: \{botong.zhu, jose guerreiro, yansheng.zhang.101, benjamin.tiller, james.windmill\}@strath.ac.uk). 
(a)

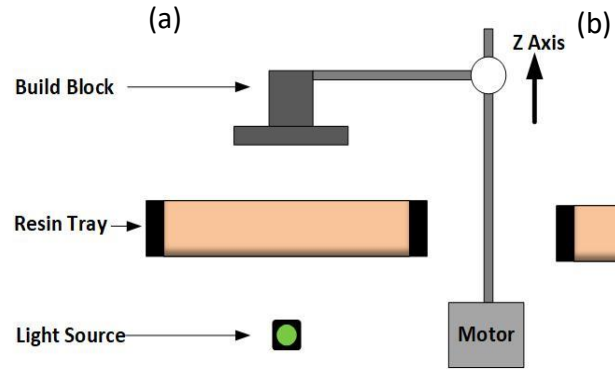

(b)

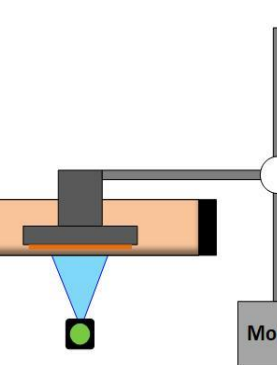

(c)

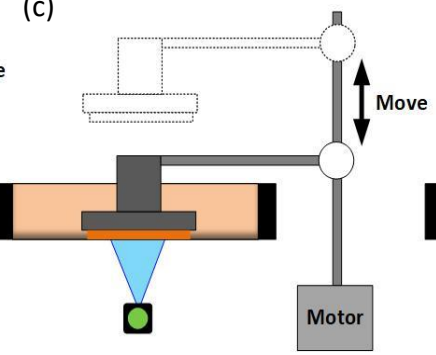

(d)

Figure 2. The workflow of the DLP printer: (a) Starting point of the 3D printing progress and main components inside the DLP printer. (b) The building block moves down and submerges into the resin tray to print the first layer. (c) The build block moves up and down to create the object layer by layer. (d) Finish printing.

\section{THEORETICAL BACKGROUND}

\section{A. Direct Light Processing (DLP) Printer}

DLP is a type of stereolithography AM technology using a liquid photopolymer resin that cures (polymerizes) under an ultraviolet light source. Figure 2 shows a schematic of the DLP $3 \mathrm{D}$ printing process. Once the $3 \mathrm{D}$ printing design slices are uploaded to the printer, the light source (DLP projector) projects the first slice image to a vat of liquid resin. Figure 2 (a) shows the starting point of the $3 \mathrm{D}$ printing process. The build block moves down and submerges into the resin tray as shown in Figure 2 (b). The exposed resin hardens on the surface of the build block to create the first layer. The block then moves up and down to print the rest of the structure layer by layer as shown in Figure 2 (c). The process is repeated until the 3D structure is completed as shown in Figure 2 (d). Then the sample is moved up from the build tray, cleaned of any extra resin, support structures are removed, and the final finished structure is ready for characterisation.

\section{B. Resin Optical Absorption}

The absorption property of the material and the related light penetration can be explained by Beer's law as shown in (1) [19], where $I(z)$ is the intensity of the light in units of $W / \mathrm{cm}^{-2}, z$ is the depth of the penetration in the resin with $z \geq 0$ (as shown in Figure 2 (a)). Hua et al. [19] proposed a mathematical model assuming the light incident from a source when the depth $z<0$ is propagating along the $+z$ direction. $I_{0}$ is the initial light intensity on the boundary between the resin and tray (when $z=$ $0) . \alpha$ is the absorbance of the resin with units of $\mu m^{-1}$.

$$
I(z)=I_{0} e^{-\alpha z}
$$

The total dose of light $D(z, t)$ [19], in units of $J / \mathrm{cm}^{-2}$ can be expressed by (2), where the $t$ is the exposure time.

$$
D(z, t)=t I(z)=t I_{0} e^{-\alpha z}
$$

Current commercial stereolithography printers and their materials work better at millimetre scale $(>1 \mathrm{~mm})$ down towards sub-millimeter scale [20], and therefore are unlikely to meet the required resolution for the fabrication of micro-scale devices. Hua Gong et.al [19], [20] proposed an improved 3D printing resin formula by adding different absorbers (dyes) into the ultraviolet (UV) curable resin in order to increase absorbance $(\alpha)$ in equation (1) and (2). This improved formula increases the resolution to $\sim 100 \mu \mathrm{m}$ using a commercial DLP printer [19] and $\sim 20 \mu \mathrm{m}$ using a custom-built DLP-SLA printer [20]. The penetration depth and exposure time need to be adjusted by adding different amounts of absorbers. In this project, the absorber was SUDAN 1 and the optimized concentration was $0.5 \%$ by weight (wt. \%).

\section{Polymer/Multiwall Carbon Nanotubes (MWCNTs) Composite}

Carbon nanotubes (CNTs) have emerged in recent years as one of the most promising nanofillers to enhance the mechanical properties (Young's modulus) [21], [22] and conductivity of polymers [23]-[25]. CNTs are long cylinders of covalently bonded carbon atoms only a few nanometers in diameter, but with lengths ca. tens of microns [24]. CNT/polymer composite based sensors have been studied for some time, but with limitations in terms of repeatability, sensitivity and stability [8]. However, those disadvantages could be improved through the use of AM technology. Researchers also indicate that beyond a certain weight fraction, which is known as the percolation threshold, of CNTs, there is a sharp rise in conductivity due to the formation of electrically conductive pathways inside the CNT/polymer composite [26]. The percolation threshold of multiwall (MW) CNTs was found to be hugely different when it is embedded in different polymer matrixes [26]. Polyethylene glycol diacrylate (PEGDA) is one of the conventional materials in stereolithography AM technology because of its UV curing properties. The conductivity of MWCNT/ PEGDA composite with the MWCNT concentration varied from $0.1 \%$ to $1.25 \%$ was previously studied [17], where it was concluded that there is a massive increase in the conductivity when the concentration increases from $0.1 \%$ to $0.5 \%$, but this growth is dramatically slowed down when the concentration is greater than $0.5 \%$. Also, several experimental trials indicate that the printability (resolution) becomes poorer when the CNT concentration is higher than $1.0 \%$ due to UV light scattering by the CNTs. Further, the composite layer must be at least $50 \mu \mathrm{m}$ thick to be conductive. Moreover, the composite resin becomes difficult to polymerize when the concentration is higher than $2 \%$. Therefore, $0.5 \%$ (wt. \%) is the optimized concentration chosen for this study. 


\section{MATERIAL, DESIGN AND FABRICATION}

\section{A. Material preparation}

A stereolithography base resin usually includes at least two elements [19]: a monomer material and a photoinitiator. If some additional functionality (e.g. conductivity, flexibility, higher resolution, or piezoelectricity) is required, then nano-fillers can be added into the base resins. Two different functional stereolithography resins were synthesized in this project by using PEGDA (MW250), phenylbis (2,4,6-trimethylbenzoyl) phosphine oxide (Irgacure 819), SUDAN 1 (S1) and MWCNT (NC7000, average diameter $9.5 \mathrm{~nm}$, average length $1.5 \mu \mathrm{m}$ ). PEGDA, Irgacure 819 and S1 were purchased from SigmaAldrich (St. Louis, MO) and the MWCNT was from Nanocyl (Sambreville, Belgium).

The first type of functional stereolithography resin used - the S1/PEGDA resin - was synthesized with PEGDA (98.5\%, wt. $\%)$, Irgacure $819(1 \%$, wt. \%), and S1 $(0.5 \%$, wt. \%). The resin was then mixed in a Thinky ARE 250 planetary mixer (Intertronics) and sonicated for at least 30 minutes before use.

The second type of functional stereolithography resin - the MWCNT/PEGDA resin - was synthesized with PEGDA $(98.5 \%$, wt. \%), Irgacure $819(1 \%$, wt. \%) and MWCNT $(0.5 \%$, wt. \%). The weighing and initial mixing process were carried out using vacuum glove bags in order to minimize any potential hazard from MWCNTs release. The mixture was then stirred for at least $24 \mathrm{~h}$ with a magnetic stirrer before use.

\section{B. Transducer Design}

A conventional capacitive transducer consists of a dielectric thin film with one side metalized, a rigid conductive backplate and an air gap between the dielectric film and the bottom electrode (as shown in Figure 1). The resonant frequency of the capacitive transducer is mainly affected by the shape, thickness, size and the intrinsic stress of the thin film. Generally, the intrinsic stress is generated from the fabrication progress. When the transducer has an edge-clamped film with low intrinsic stress the influence from the intrinsic stress is ignored, and the resonant frequency of the thin circular plate $\left(f_{t p}\right)$ can be expressed by (3) \& (4) [27], where $K_{n}$ is a constant that takes the value 10.22 for a clamped circular thin film in the fundamental resonance mode, $R$ is the radius of the thin plate, $D_{E}$ is the flexural rigidity of the circular thin plate, $h$ is the thickness of the thin film, $\rho$ is the density of the thin plate, $E$ is the Young's modulus and $\mu$ is the Poisson ratio. When the intrinsic stress is large enough, the resonant frequency of the thin plate $\left(f_{t p}\right)$ is dominated by the intrinsic stress $(T)$ rather than by the flexural rigidity (as shown in (5)). The squeeze film effect occurs when the air gap (depth of cavity) is very narrow [28]. Squeeze film damping becomes more important than the drag force damping of air if the thickness of the gas gap is smaller than one-third of the thickness of the plate [28]. Even so, the air gap in a design should be thin enough to maximize sensitivity. Limited by the printing resolution of this project, the air gap of the transducer presented here is slightly thicker than the thin film. Therefore, the squeeze film effect is not significant in this transducer design. However, the design does include some releasing holes to minimize squeeze film damping.

$$
\begin{aligned}
& f_{t p}=\frac{K_{n}}{2 \pi R^{2}} \sqrt{\frac{D_{E}}{\rho h}} \\
& D_{E}=\frac{E h^{3}}{12\left(1-\mu^{2}\right)} \\
& f_{t p}=\frac{K_{n}}{2 \pi R^{2}} \sqrt{\frac{T}{\rho h}}
\end{aligned}
$$

The computer designed 3D model was produced as shown in Figure 3(a). The anchor and the backplate (orange parts) are made by a passive polymer which is synthesized with PEGDA and Sudan 1, the black layer between air-gap and the backplate is the PEGDA/MWCNT composite polymer. The top silver round layer is an aluminate electrode. The transducer design used three diameters, chosen to be $1.8 \mathrm{~mm}, 2.1 \mathrm{~mm}$ and $3.2 \mathrm{~mm}$, in order to place resonant frequencies at $19 \mathrm{kHz}$, $35 \mathrm{kHz}$ and $54 \mathrm{kHz}$ respectively. After testing the accuracy of fabrication, the height of the air gap was determined to be $\sim 120 \mu \mathrm{m}$ and the thickness of the dielectric film was $\sim 80 \mu \mathrm{m}$. The thickness of the top aluminum electrode was $0.11 \mu \mathrm{m}$ deposited by using a thin film deposition system (E306, Edwards). The thickness of the bottom MWCNT electrode is $\sim 50 \mu \mathrm{m}$. Simulating the resonant frequency of the capacitive transducer requires knowledge of the mechanical properties of the S1/PEGDA composite. This depends on any variations in the exposure time and the concentration of the absorber during the AM. The estimated mechanical properties utilized in this work (Young's Modules $E=1 \mathrm{GPa}$, Poisson Ratio $\mu=0.32$ and Density $\rho=1183 \mathrm{~kg} / \mathrm{m}^{3}$ ) are based on a previous study [17]. Figure 3 (b) shows the cross-sectional X-ray computerized tomography (CT) scan of the transducer. The aluminum electrode cannot be replaced by a 3D printed MWCNT/PEGDA layer since the limitations of the AM resolution and the conductivity in this study mean that such a layer would be too thick to vibrate with incident acoustic energy. (a)

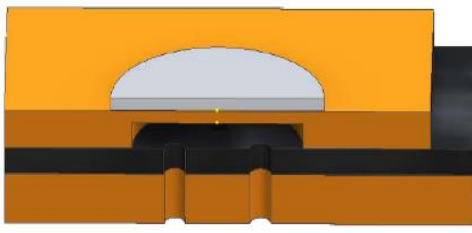

(b)

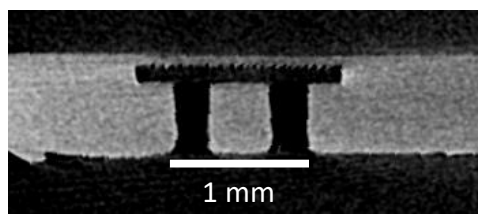

Aluminide

S1/PEGDA

MWCNT/PEGDA
Figure 3. The cross-sectional view of an AM capacitive acoustic transducer: (a) 3D computer model and (b) X-ray CT scan. 
(a)

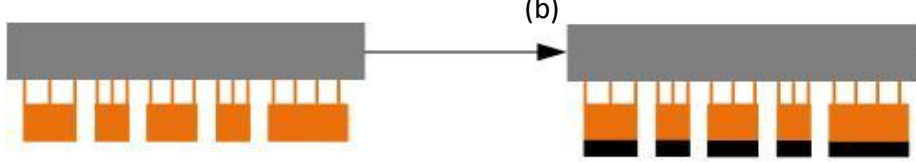

(g)

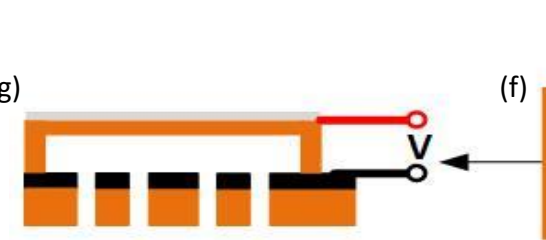

Aluminum

S1/PEGDA

Deposit Aluminum Electrode

Figure 4. The fabrication flow for the capacitive transducer: (a) Print S1/PEGDA backplate with releasing holes. (b) Print MWCNT/PEGDA conductive layer with releasing holes as the bottom electrode. (c) Print S1/PEGDA air gap and dielectric thin film. (d) Print S1/PEGDA mask for top electrode coating. (e) Flip the transducer and mount the mask. (f) Deposit aluminium electrode on the top of the film as the top electrode. (g) Connect wires to the electrodes.

\section{Fabrication}

An ASIGA stereolithography printer (PICO 2 HD $27 \mu m$ ) was utilized to fabricate the capacitive transducer. This printer has a $27 \mu m$ pixel size in the $\mathrm{X}-\mathrm{Y}$ plane and $1 \mu \mathrm{m}$ available resolution in the $\mathrm{Z}$ axis. The wavelength of the $\mathrm{UV}$ light source is $385 \mathrm{~nm}$. A build layer of $10 \mu \mathrm{m}$ and exposure time of $3.6 s$ allow an optimized resolution for the material formula introduced above. Figure 4 shows the workflow for the transducer's fabrication process. First of all, the S1/PEGDA backplate with support is printed on the building block. Then, any extra unpolymerized resin is cleaned away with Ethanol, and the system is manually swapped to a MWCNT/PEGDA resin tray to print the conductive bottom electrode layer. After that, the sample is cleaned again and the system is swapped to the S1/PEGDA build tray to print the air gap and dielectric thin film. The top electrode is final deposited. A mask is printed after the transducer sample is removed from the build tray. The mask is mounted on the flipped transducer, and then the sample is placed into the thin film deposition system where the aluminum electrode is deposited on the top of the dielectric thin film. Finally, the mask is removed and wires connected to the device's top and bottom electrodes.

\section{RESULTS}

Figure 5(a) \& (b) show the experimental setup for characterizing the transducer in sensing and transmitting modes, respectively. In Figure 5 (a), a function generator produced a 7 cycles tone-burst to drive a loudspeaker. The transducer was placed $20 \mathrm{~cm}$ away from the loudspeaker to avoid any near-field effects. A voltage supply provided different DC voltages from $0-150 \mathrm{~V}$ to bias the transducer. The electrical output signal generated by the transducer was (a)

(d)

(c)

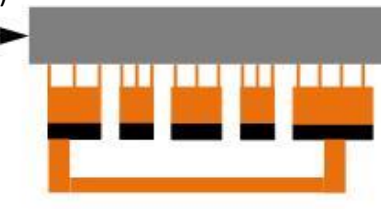

(e)

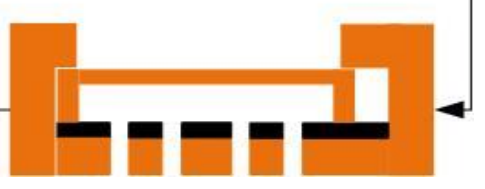

Build Block in 3D Printer

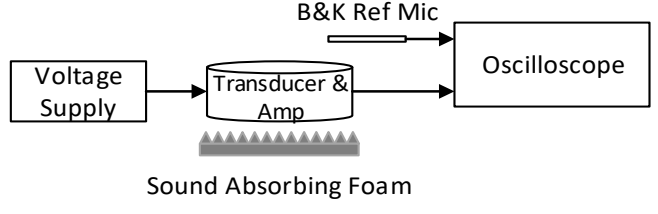

(b)

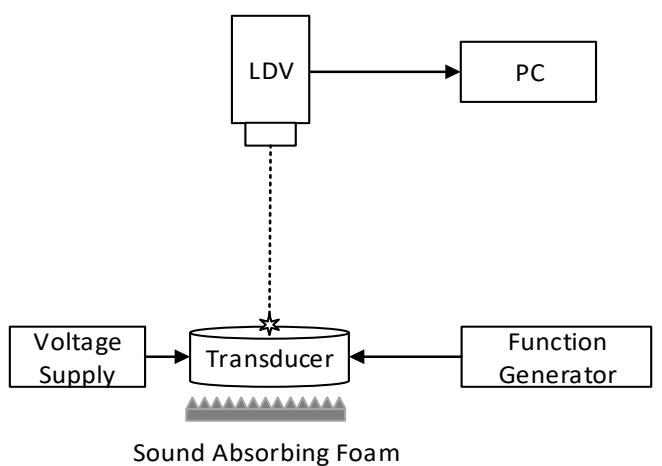

Figure 5. Simplified schematic of the experimental setup for the (a) sensing and (b) transmitting measurements.

amplified by a custom-built electronic circuit and finally acquired using an oscilloscope. A $1 / 8$ inch reference microphone (Brüel and Kjær, Type 4138) with an operational frequency range from $1 \mathrm{~Hz}$ to $110 \mathrm{kHz}$ and a paired charge 
(a)

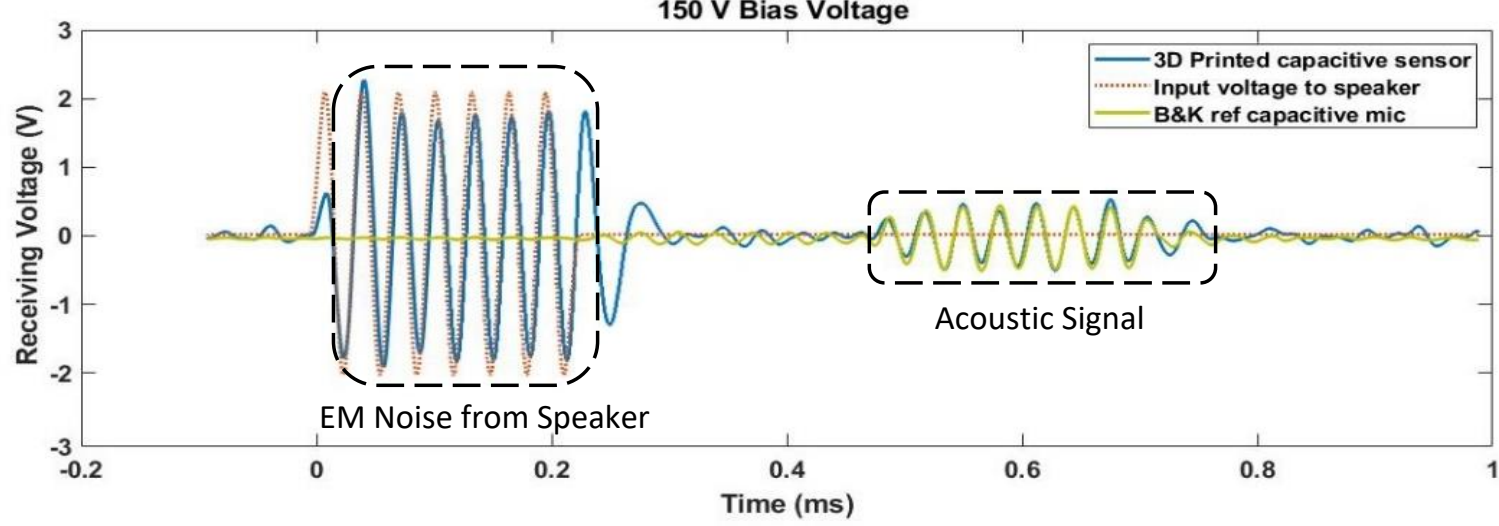

(b)

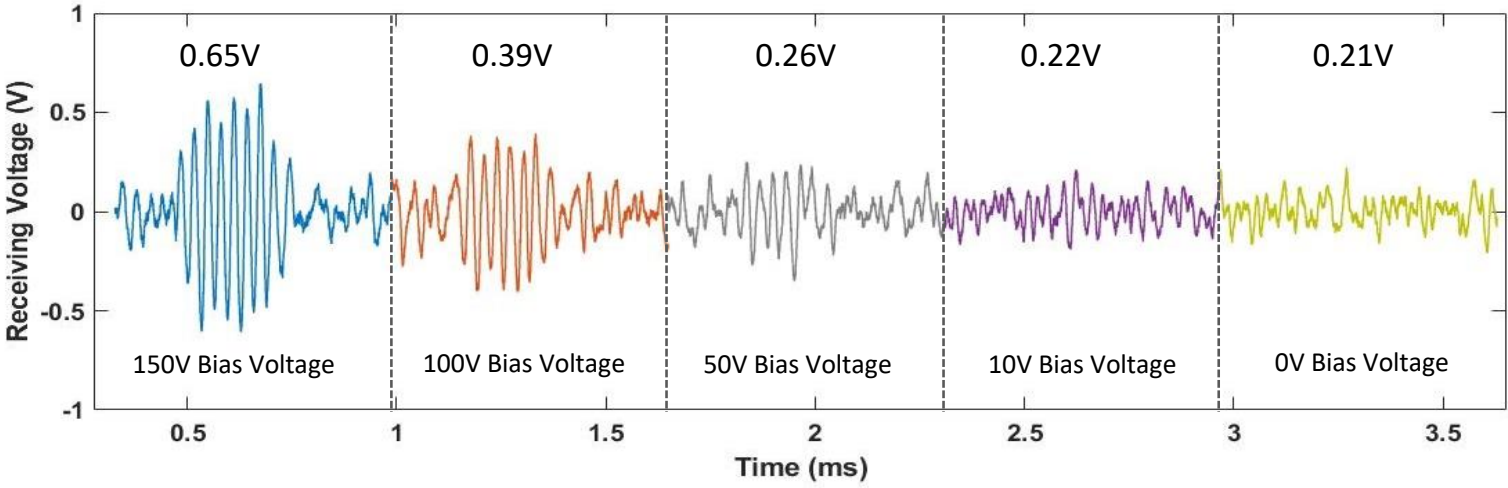

Figure 6. The electrical output time domain signal from the transducer (diameter $=2.1 \mathrm{~mm}$ ) integrated with the amplification circuit when the loudspeaker played a 7 cycles tone-burst at $35 \mathrm{kHz}$. (a) Shows the electrode magnetic and acoustic signal received by both of the 3D printed transducer and the B\&K reference microphone under $150 \mathrm{~V}$ bias voltage. (b) Shows the maximum amplitude change of the acoustic signal when providing different bias voltages from 150-0 V.

amplifier (Brüel and Kjær, type 2692) were used to measure the reference sound pressure around the transducer. The reference signal was then utilized to calibrate the sensitivity of our transducers. Sound absorbing foam was placed under the transducer and the $\mathrm{B} \& \mathrm{~K}$ microphone to avoid acoustic reflections. In Figure 5 (b), the function generator inputted a $10 \mathrm{~V}$ chirp signal to drive the transducer into vibration which was detected by a 3D Laser Doppler Vibrometer (LDV: MSA100-3D, Polytec, Inc., Waldbronn, Germany). A voltage supply was used to charge the capacitive transducer with varied DC voltage from $0-150 \mathrm{~V}$.

Table 1 . The receiving sensitivity and the static capacitance of the AM transducers with different film diameter measured with $150 \mathrm{~V}$ bias voltage at each resonant frequencies

\begin{tabular}{|l|c|c|c|}
\hline Diameter & $\mathbf{1 . 8} \mathbf{~ m m}$ & $\mathbf{2 . 1} \mathbf{~ m m}$ & $\mathbf{3 . 2} \mathbf{~ m m}$ \\
\hline Frequency & $54 \mathrm{kHz}$ & $35 \mathrm{kHz}$ & $19 \mathrm{kHz}$ \\
\hline Capacitance & $2.08 \mathrm{pF}$ & $3.74 \mathrm{pF}$ & $4.62 \mathrm{pF}$ \\
\hline Sensitivity & $0.009 \mathrm{mV} / \mathrm{Pa}$ & $0.013 \mathrm{mV} / \mathrm{Pa}$ & $0.4 \mathrm{mV} / \mathrm{Pa}$ \\
\hline
\end{tabular}

Table 1 gives the value of capacitance and receiving sensitivity with respect to different membrane diameters. The static capacitance of the transducer was measured by using a 4194A Impedance/Gain-Phase Analyzer under a $150 \mathrm{~V}$ bias voltage. It is found that the capacitance of the transducer is proportional to its size. The receiving sensitivity $(S)$ of the transducers was calculated by (6) with the unit $m V / P a$ where the Output $(O)$ is the maximum amplitude of the amplified signal at the resonant frequency of the transducer, the Acoustic Pressure $(A P)$ is the sound pressure level around the transducer measured by the reference $\mathrm{B} \& \mathrm{~K}$ microphone and the Amplifier Gain $(A G)$ is the gain of our custom-built amplifier.

$$
S(m V / P a)=\frac{O(m V)}{A P(P a) \times A G}
$$

The receiving sensitivity of the AM capacitive transducer can reach up to $0.4 \mathrm{mV} / \mathrm{Pa}$ before amplification (as shown in Figure 6), however, the receiving sensitivity rapidly decreases to $0.009 \mathrm{mV} / \mathrm{Pa}$ when the diameter of the transducers decreases. The reason for this is that the smaller active area results in lower sensing energy and smaller capacitance change [29]. Figure 6 (a) also shows that the acoustic signal received by the AM transducer and the reference $\mathrm{B} \& \mathrm{~K}$ microphone are very similar when both devices are operating at the resonant frequency of the AM transducer, nevertheless, this AM transducer set-up has less immunity to electromagnetic interference due to the absence of any shielding or proper packaging design. Similar to other capacitive transducers (as shown in Figure 6 (b)), this AM capacitive transducer requires a bias voltage to operate, and its sensitivity increases proportionally when providing higher bias voltages. The transmitting mode of the AM transducer was characterized by measuring the vibration of the thin film as shown in Figure 5 (b) as the transmitted sound field was too weak to be measured 
by the reference microphone. Figure 7(a) shows the frequency response of the transducer with $2.1 \mathrm{~mm}$ diameter. The output signal increases in amplitude when providing a larger bias voltage. Figure 7 (b) shows that the resonant frequency of the transducer with different diameters $(1.8 \mathrm{~mm}, 2.1 \mathrm{~mm}$ and $3.2 \mathrm{~mm}$ ) was characterized at $54 \mathrm{kHz}, 35 \mathrm{kHz}$ and $19 \mathrm{kHz}$ respectively which can be matched with the simulated resonant frequency curve. The simulation method assumes the intrinsic stress is low and the mechanical properties used in both methods are given in the transducer design section. Figure 8(a) shows the AM transducer integrated with a custom-built amplifying circuit. The circuit board is $3 \mathrm{~cm} \times 4 \mathrm{~cm}$, and the silver part is the active area of the transducer. A hybrid JFET operational amplifier circuit was built in order to amplify the signals captured by the 3D printed sensor, as illustrated in Figure 8 (b) [30]. This circuit was designed with a constant gain of $50 \mathrm{~dB}$ between $1-100 \mathrm{kHz}$. The experiment is for 3 one-off samples. Assuming the AM technology will cause a 5\% variation of the diameter size of the transducer arising from polymer shrinkage [31], the estimated resonance variability as calculated from Equations (3) and (4) at $1.8 \mathrm{~mm}, 2.1 \mathrm{~mm}$ and $3.2 \mathrm{~mm}$ diameter transducer are $45 \pm 5.8 \mathrm{kHz}, 33 \pm$ $3.6 \mathrm{kHz}$ and $14 \pm 1.5 \mathrm{kHz}$, respectively.

(a)

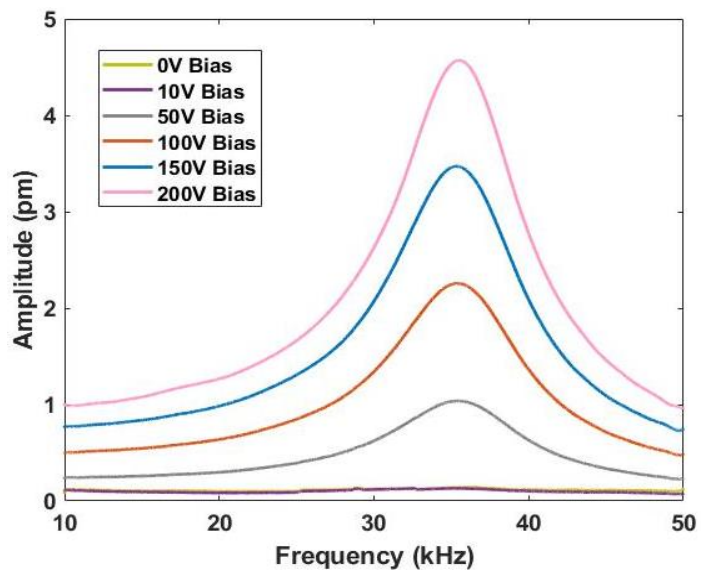

(b)

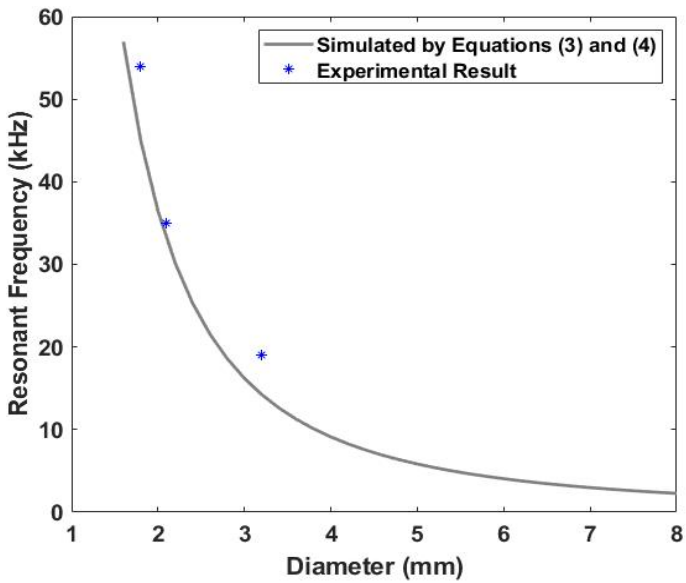

Figure $\%$. (a) I he trequency response of the transducer (diameter $=2.1 \mathrm{~mm}$ ) was measured by LDV when the transducer was driven by $10 \mathrm{~V}$ chirp signal and different bias voltages. (b) The comparison between the simulated and experimental resonant frequency. The simulation assumes that the intrinsic stress is low. (a)

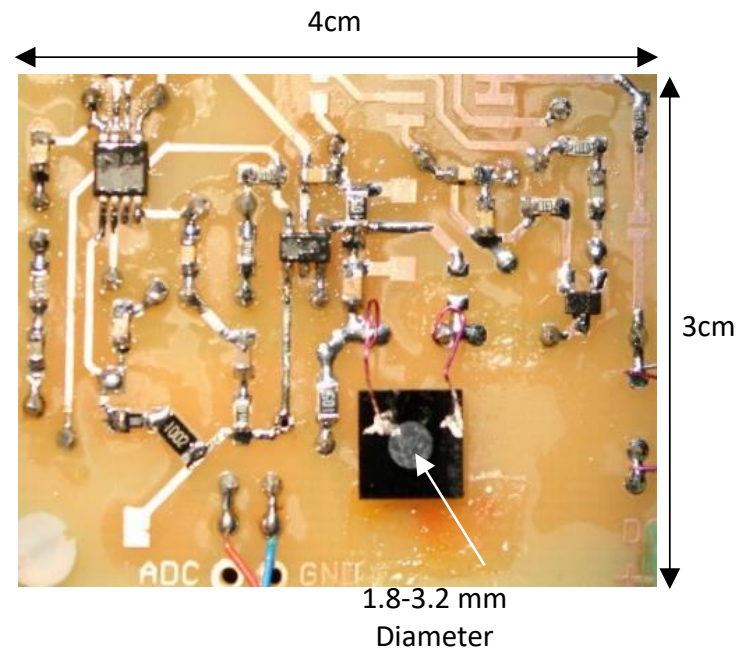

(b)

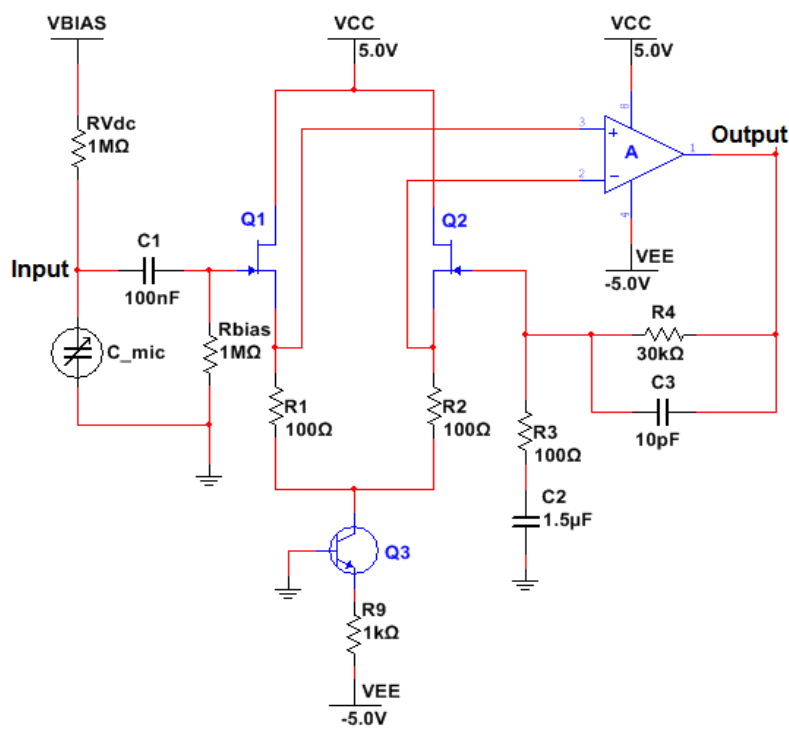

Figure 8. (a) Custom-built amplifying circuit integrated with the AM transducer. The size of the circuit board is $3 \mathrm{~cm} \times 4 \mathrm{~cm}$, and the silver part with different diameters $(1.8-3.2 \mathrm{~mm})$ is the active area of the transducer. (b) Schematic of amplifying circuit. The custom-built amplifier provides a constant gain of approximately $50 d B$ with operational bandwidth ( $-3 d B$ bandwidth) of $1-100 \mathrm{kHz}$

\section{CONCLUSION}

This paper demonstrates the first micron-scale capacitive transducer fabricated with a commercial DLP 3D printer. Transducers with different size have been fabricated and integrated with electronics to characterize the overall performance. The characterization results indicate that the performance of the AM transducer in sensing mode can reach up to $0.4 \mathrm{mV} / \mathrm{Pa}$ at its resonant frequency. In contrast, the receiving open circuit sensitivity of a circular-clamped MEMS microphone and the commercial B\&K microphone (Type 4138) with similar size are reported to be $\sim 1 \mathrm{mV} / \mathrm{Pa}$ at a bias voltage of $1 \mathrm{~V}$ [4] and $1 \mathrm{mV} / \mathrm{Pa}$ at a bias voltage of $200 \mathrm{~V}$ [32], respectively. The collapse voltage of conventional MEMS microphones is usually lower than $5 \mathrm{~V}$ due to their narrow air gaps $(\sim 2 \mu \mathrm{m})$ [2]. The required bias voltage of the AM transducers is $150 \mathrm{~V}$, which is much higher than the 
conventional MEMS microphone $(<5 \mathrm{~V})$ but lower than the B\&K commercial microphone $(200 \mathrm{~V})$. Different diameters of transducer were fabricated and tested in this research study. The resonant frequency of the transducers decreases from $52 \mathrm{kHz}$ to $19 \mathrm{kHz}$ and the received sensitivity increases from $0.009 \mathrm{mV} / \mathrm{Pa}$ to $0.4 \mathrm{mV} / \mathrm{Pa}$, respectively, when the diameter of the transducers increases from $1.8 \mathrm{~mm}$ to $3.2 \mathrm{~mm}$. The current AM transducers have better performance in sensing rather than transmitting due to their low transmitting sensitivity. Because of the limitations of the commercial AM printer used, the fabrication process still requires human interaction, for example, manually swapping the resin tray, post processing the AM samples and evaporation coating the top electrode. The AM fabricating process will not take longer than two hours and the speed of coating process depends on the technique. The yield rate of the AM transducer is lower than $30 \%$ when the diameter is smaller than $1.8 \mathrm{~mm}$. The AM transducers remained functional for several months before the manuscript was submitted.

Due to the advantages of using the AM technology to create complex 3D geometry, there are possible applications for the AM transducers including integration with hearing prostheses, bio-inspired transducers with complex 3D structures, underwater sonar, and non-destructive testing (NDT). The maximum operating frequency achieved by these purpose-built AM transducers is limited by the printing resolution of the printer. However, recent developments in two-photon polymerization (TPP) can achieve a resolution below $200 \mathrm{~nm}$ [33] which could provide for a reduction in the transducer size and thus lead to operation in higher frequency ranges. Nevertheless, the performance of the transducer might be further improved by decreasing the air gap, and also by increasing the conductivity of the MWCNT/PEGDA composite as a result of optimizing the formula and exposure time. Further to this, recent work has demonstrated AM fabrication with multiple materials automatically by using a custom-build 3D printer [34]. The fabrication process of AM capacitive transducers could therefore become far more automatic in the future.

\section{ACKNOWLEDGMENT}

This work was supported by the EPSRC under grants EP/L022125/1 and EP/M026701/1, and by the European Research Council under the European Union's Seventh Framework Programme (FP/2007-2013)/ERC Grant Agreement n. [615030].

\section{REFERENCES}

[1] P. R. Scheeper et al., "A New Measurement Microphone Based on MEMS Technology," vol. 12, no. 6, pp. 880-891, 2003.

[2] P. R. Scheepera, A. G. . Van der Donk, W. Olthuis, and P. Bergveld, "A review of silicon microphones," Sensors Actuators A, vol. 44, pp. 1-11, 1994.

[3] J. Liu, D. T. Martin, T. Nishida, L. N. Cattafesta, M. Sheplak, and B. P. Mann, "Harmonic balance nonlinear identification of a capacitive dual-backplate MEMS microphone," J. Microelectromechanical
Syst., vol. 17, no. 3, pp. 698-708, 2008.

[4] M. Füldner, A. Dehé, and R. Lerch, "Analytical analysis and finite element simulation of advanced membranes for silicon microphones," IEEE Sens. J., vol. 5, no. 5, pp. 857-862, 2005.

[5] P. Mattila, F. Tsuzuki, and V. Heli, "Electroacoustic Model for Electrostatic Ultrasonic Transducers with V-Grooved Backplates," vol. 42, no. 1, pp. 1-7, 1995.

[6] B. Zhu, B. Tiller, A. J. Walker, A. J. Mulholland, and J. F. C. Windmill, “'Pipe Organ’ Inspired Air-coupled Ultrasonic Transducers with Broader Bandwidth," IEEE Trans. Ultrason. Ferroelectr. Freq. Control, pp. 1-10, 2018.

[7] R. N. Miles and R. R. Hoy, "The development of a biologically-inspired directional microphone for hearing aids," Audiol. Neurotol., vol. 11, no. 2, pp. 86-94, 2005.

[8] M. O. F. Emon and J. W. Choi, "Flexible piezoresistive sensors embedded in 3D printed tires," Sensors (Switzerland), vol. 17, no. 3, p. 656, 2017.

[9] K. Kim, W. Zhu, X. Qu, C. Aaronson, and W. R. Mccall, "3D Optical Printing of Piezoelectric Materials," ACS Nano, vol. 8, no. 10, pp. 9799-9806, 2014.

[10] R. Domingo-Roca, J. C. Jackson, and J. F. C. Windmill, "Bioinspired 3D-printed piezoelectric device for acoustic frequency separation," Sensors Actuators A Phys., vol. 271, pp. 1-8, 2018.

[11] J. H. Jung et al., "Lead-free NaNbO 3 nanowires for a high output piezoelectric nanogenerator," ACS Nano, vol. 5, no. 12, pp. 10041-10046, 2011.

[12] A. Zolfagharian, A. Z. Kouzani, S. Y. Khoo, A. A. A. Moghadam, I. Gibson, and A. Kaynak, "Evolution of 3D printed soft actuators," Sensors Actuators, A Phys., vol. 250, pp. 258-272, 2016.

[13] A. Miriyev, K. Stack, and H. Lipson, "Soft material for soft actuators," Nat. Commun., vol. 8, no. 1, p. 596, 2017.

[14] C. Liu et al., "3D Printing Technologies for Flexible Tactile Sensors toward Wearable Electronics and Electronic Skin,” Polymers (Basel)., vol. 10, no. 6, pp. 1-31, 2018.

[15] M. S. Mannoor et al., "3D Printed Bionic Ears," NANO Lett, no. 13, pp. 2634-2639, 2013.

[16] R. Haque, E. Ogam, P. Benaben, and X. Boddaert, "Inkjet-Printed Membrane for a Capacitive Acoustic Sensor: Development and Characterization Using Laser Vibrometer," Sensors, vol. 17, no. 5, p. 1056, 2017.

[17] B. Tiller et al., "Piezoelectric microphone via a digital light processing 3D printing process," Mater. Des., vol. 165, pp. 1-27, 2019.

[18] Y. Zhang, J. F. C. Windmill, and D. Uttamchandani, "Biomimetic MEMS directional microphone structures for multi-band operation," IEEE SENSORS 2014 Proc., pp. 440-443, 2014.

[19] H. Gong, M. Beauchamp, S. Perry, T. Woolley, and G P. Nordin, "Optical approach to resin formulation for 3D printed microfluidic," RSC Adv., vol. 11, no. 5, pp. 106621-106632, 2015. 
[20] H. Gong, B. P. Bickham, A. T. Woolley, and G. P. Nordin, "Custom 3D printer and resin for $18 \mu \mathrm{m} \times 20$ $\mu \mathrm{m}$ microfluidic flow channels," vol. 17, no. 17, 2017.

[21] D. Qian, E. C. Dickey, R. Andrews, and T. Rantell, "Load transfer and deformation mechanisms in carbon nanotube-polystyrene composites," Appl. Phys. Lett., vol. 76, no. 20, pp. 2868-2870, 2000.

[22] M. J. Biercuk, M. C. Llaguno, M. Radosavljevic, J. K. Hyun, A. T. Johnson, and J. E. Fischer, "Carbon nanotube composites for thermal management," Appl. Phys. Lett., vol. 80, no. 15, pp. 2767-2769, 2002.

[23] H. Ishikawa, S. Fudetani, and M. Hirohashi, "Mechanical properties of thin films measured by nanoindenters," Appl. Surf. Sci., vol. 178, pp. 56-62, 2001.

[24] V. Choudhary and A. Gupta, "Polymer/Carbon Nanotube Nanocomposites," in Carbon Nanotubes Polymer Nanocomposites, 2011, pp. 66-90.

[25] B. Kracke and B. Damaschke, "Measurement of nanohardness and nanoelasticity of thin gold films with scanning force microscope," Appl. Phys. Lett., vol. 77, no. 3, pp. 361-363, 2000.

[26] W. Bauhofer, J. Z. Kovacs, T. Swan, T. Swan, and T. Swan, "A review and analysis of electrical percolation in carbon nanotube polymer composites," Compos. Sci. Technol., vol. 69, no. 10, pp. 1486-1498, 2009.

[27] W. C. Young and R. G. Budynas, Roark's Formulas for Stress and Strain. 2017.

[28] M. Bao and H. Yang, "Squeeze film air damping in MEMS," Sensors Actuators, A Phys., vol. 136, no. 1, pp. 3-27, 2007.

[29] A. Dehé, M. Wurzer, M. Füldner, U. Krumbein, and I. T. Ag, "The Infineon Silicon MEMS Microphone," IEEE Sens. J., vol. 15, no. 3, pp. 95-99, 2013.

[30] Alexandrov G and C. N., Some Tips on Making a FETching Discrete Amplifier. Analog Dialogue, 2013.

[31] B. Zhu, B. Tiller, J. F. C. Windmill, A. J. Mulholland, and A. J. Walker, "“ Pipe Organ' Air -coupled Broad Bandwidth Transducer," in IEEE Int. Ultrason. Symp. IUS, 2017, pp. 1-4.

[32] Brüel \& Kjær, "Product Data: 1/8" Pressure - field Microphone Type 4138," Datasheet. .

[33] J. Serbin, A. Egbert, A. Ostendorf, and B. N. Chichkov, "Femtosecond laser-induced two-photon polymerization of inorganic - organic hybrid materials for applications in photonics," vol. 28, no. 5, pp. 301303, 2003.

[34] M. Vaezi, S. Chianrabutra, B. Mellor, and S. Yang, "Multiple material additive manufacturing - Part 1: a review," Virtual Phys. Prototyp., vol. 8, no. 1, pp. 1950, 2013.

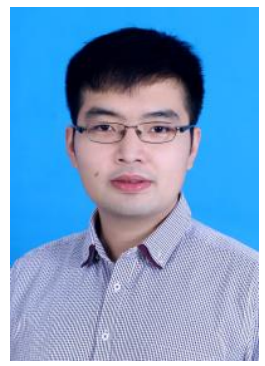

Botong Zhu received the bachelor's degree from the University of Strathclyde in 2015, where he is currently pursuing the Ph.D. degree with the Department of Electronic and Electrical Engineering. He is also a PhD student in EPSRC Centre for Doctoral Training in Quantitative NDE. His research interests are in the field of air- coupled transducer development and novel additive manufacturing technologies.

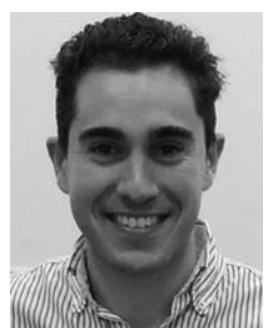

José Guerreiro (S'15-M'18) was born in Lisbon, Portugal, in 1988. He received the B.S. and M.S. degrees in electronic and telecommunications engineering from the High Institute of Engineering of Lisbon, Lisbon, Portugal, in 2013, and he is currently working toward the Ph.D. degree in electronic and electrical engineering at the University of Strathclyde, Glasgow,

U.K.

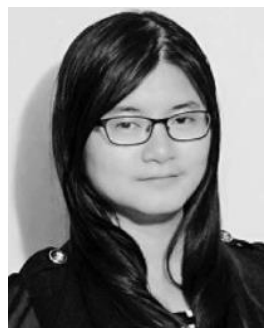

Yansheng Zhang received the bachelor's degree from the University of Strathclyde in 2012, where she is currently pursuing the Ph.D. degree with the Department of Electronic and Electrical Engineering. She is currently a Research Assistant with the Department of Electronic and Electrical Engineering, University of Strathclyde. Her research interests are in the field of biologically-inspired MEMS microphone development.

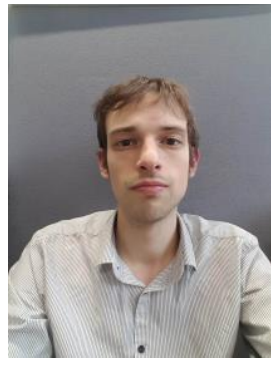

Benjamin $\mathbf{P}$ Tiller is a Research Associate at the University of Strathclyde, in the Department of Electronics and Electrical Engineering, Glasgow, United Kingdom. He did his PhD at University of Glasgow focusing on the frequency scaling relationship of acoustic streaming in microfluidic environments. Before that he completed an MSc in Physics with Theoretical Physics at University of Nottingham. His current research interests involve developing novel 3D printed techniques that enable new sensing technologies.

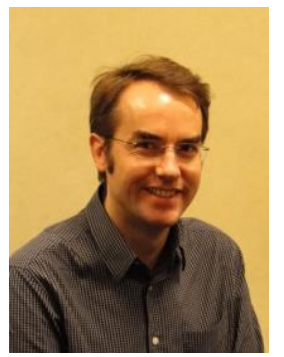

James F. C. Windmill (M'99-SM'18) is a Professor in the Department of Electronic and Electrical Engineering at the University of Strathclyde, Glasgow, United Kingdom. He received a B.Eng. degree in electronic engineering from the University of Plymouth, UK, in 1998 and a Ph.D. degree in magnetic microscopy from University of Plymouth, UK, in 2002. He joined the Centre for Ultrasonic Engineering (CUE) at the University of Strathclyde as a lecturer in 2008. He has over 18 years of research and development experience in the areas of sensors and hearing systems. His research interests are in the field of biologically-inspired acoustic systems, from the fundamental biology to various engineering application topics. 